\title{
La conformación del nuevo espacio público en la zona metropolitana de Poza Rica
}

\author{
Blanca Inés Aguilar Frías a*, Jesús Martínez Bocardi a , Héctor Rivera Torres a , Carlos César Morales Guzmán ${ }^{\text {a }}$, Calixto \\ Martínez Cruz ${ }^{\mathrm{a}}$ \\ ${ }^{a}$ Facultad de Arquitectura, UV, Carretera Poza Rica-Papantla, Poza Rica, Veracruz, México
}

\begin{abstract}
Resumen
La ciudad extensa genera compromisos en la satisfacción de equipamiento e infraestructura y en cuanto a los espacios públicos y privados. La Zona Metropolitana de Poza Rica (ZMPR) en su municipio central, el parque urbano, como equipamiento para el sano desarrollo de los citadinos modernos, se revela como un andamio para la revitalización de la vida comunitaria ávida en su atención sobre este tipo de espacios considerablemente escasos y con deficiente accesibilidad al paso del tiempo. Las urbes modernas con los espacios públicos intervenidos se posicionan como nodos de interconexión entre los puntos centrales de la misma conciliando con ellos la integración al entramado urbano necesario para la vivificación y consolidación dando carácter e identidad a cada zona. El damero de la ciudad cuenta con zonas grises en las colonias, áreas escolares y en vialidades identificadas con potencial de utilización para estos espacios urbano con fuerte participación ciudadana al realizarse acciones que inmiscuya a niños, adolescentes y adultos mayores para la concientización de principios de accesibilidad universal y diseño sustentable para espacios públicos seguros. El parque urbano como espacio de expresión social alberga la colectividad diaria, la memoria colectiva transformándose de ser considerada solo como el espacio paisajístico por excelencia dando paso a la convivencia y tránsito modal. Es precisamente los espacios residuales donde prima la consideración de diseño sostenible del nuevo espacio público.
\end{abstract}

Palabras Clave: Espacio público, accesibilidad universal, sustentabilidad, ciudad extensa, zona metropolitana.

\section{Introducción}

Con Las urbes modernas, en sus espacios públicos, se posicionan como nodos de interconexión entre los puntos centrales de la misma conciliando con ellos la integración al entramado urbano necesario para la vivificación y consolidación, dando con ello carácter e identidad a cada zona.

El damero de la ciudad cuenta con zonas en desuso en las colonias, áreas escolares y en vialidades con potencial de utilización para estos espacios urbanos con fuerte participación ciudadana al realizarse acciones que inmiscuya a niños, adolescentes y adultos mayores para la concientización en uso de lineamientos de principios de accesibilidad universal y diseño sustentable para espacios públicos seguros.

El parque urbano como espacio de expresión social alberga la colectividad diaria, la memoria colectiva transformándose de ser considerada solo como el espacio paisajístico por excelencia a un lugar de convivencia social y tránsito modal. El ser humano requiere de espacios para realizar sus actividades cotidianas y de espacios para descansar de sus mismas actividades cotidianas, sea en casa o en la ciudad. Los espacios excedentes en las ciudades son fuente de la posible revitalización de las jornadas dinámicas y, muy probablemente, enlace para la movilidad urbana sustentable. Para la zona de estudio, se dará prioridad a los espacios residuales y en esterilidad para la aplicación del diseño sustentable del espacio público.

Las expresiones artísticas favorecen la dinamización visual que conlleva a espacios de encuentros agradables. Los parques urbanos integradores reúnen aspectos como lo son la conceptualización arquitectónica social revalorizada, la identidad sincrónica entre el ciudadano y su espacio recreativo, así como la sustentabilidad que toca a estos espacios desde conceptualización y delimitación.

La caracterización del nuevo espacio público urbano apoyará la concreción edilicia de parques públicos de la ZMPR. Por tanto, se entiende como área recreativa aquella equipada con juegos mecánicos o manuales, empleadas generalmente para la diversión de los niños, mientras que como áreas deportivas a los espacios acondicionados para la práctica de algún deporte, como puede ser campos para jugar básquetbol, voleibol, etc. (INEGI, 2007).

\section{Materiales y Métodos}

El presente artículo es la continuidad de la investigación que sobre el "Parque urbano revitalizante" se iniciara como tópico de investigación dentro Observatorio Urbano y Arquitectura Sustentable (OUrbe) y el Cuerpo Académico 346 Arquitectura y Urbanismo (UV-CA-346), y para esta ocasión se presenta un avance. Corresponde a una visión de integración del espacio público, planificado o no, dentro de la Zona Metropolitana de Poza Rica (ZMPR).

El espacio público ha sido el eterno compañero de la ciudad, desde lugar de la vida pública de la polis griega hasta el uso colectivo de expresión de las ideas, del colectivo cultural, por lo tanto, es el espacio con definición física, cultural e ideológico político; física por su dimensión tangible, cultural por lo que respecta a la expresión e ideológico político por lo representativo a la manifestación del colectivo.

\footnotetext{
* Autor en correspondencia.

Correos electrónicos: bagui1ar@uv.mx (Dra. Blanca Inés Aguilar Frías), arquitecturahoy@out7ook. es (Facultad de Arquitectura),
} 
Con estos tres elementos se establece el tipo de espacio público, por ejemplo, en la periferia urbana con su crecimiento acelerado y desordenado, donde se cuenta con espacios en vacío "con forma", tal como Raquel Perahia comenta, esclareciendo que no es un lugar solitario sino que está avecindado con edificios posiblemente consolidados, vialidades primarias o secundarias (Perahia, 2007), en este caso se puede identificar el parque infantil en la colonia El Mollejón. En cuanto a otra etapa de la investigación, se revisó bibliografía relativa al tema separando los tópicos sustanciales al espacio público enmarcado con la normatividad y lineamientos de diseño sustentable existente en el país e internacional, tanto para desarrollo urbano como regional, debido a contar dentro del perímetro de la ZMPR con un alto porcentaje de territorio rural.

Con estos tres elementos se establece el tipo de espacio público, por ejemplo, en la periferia urbana con su crecimiento acelerado y desordenado, donde se cuenta con espacios en vacío "con forma", tal como Raquel Perahia comenta, esclareciendo que no es un lugar solitario, sino que está avecindado con edificios

\subsection{Descripción del área de estudio}

El INEGI, CONAPO y la Secretaría de Desarrollo Social ha reconocido la existencia de 8 zonas metropolitanas en Veracruz: ZM Veracruz, ZM Xalapa, ZM Poza Rica, ZM Orizaba, ZM Minatitlán, ZM Coatzacoalcos, ZM Córdoba, ZM Acayucan. La tercera de ellas es la Zona Metropolitana de Poza Rica que está conformada por 5 municipios Coatzintla, Cazones, Papantla, Poza Rica y Tihuatlán, con una población total de 481 mil 389 habitantes.

Como se aprecia en la figura 1, Poza Rica, el municipio central de la ZMPR, se ubica al norte del estado de Veracruz, es el municipio con menor extensión territorial pero con la mayor cantidad de población de todos los que la conforman. Estableciendo la zona observada se enfoca a la conceptualización del tema que nos ocupa, el espacio público. Identificamos al espacio público urbano como aquel terreno de la ciudad donde tiene derecho estar, disfrutar, circular o pasar todo ciudadano, y dentro de estos espacios se diferencia aquellos que son abiertos o cerrados, como los jardines, parques, plazas para los del primer grupo, bibliotecas públicas y centros comunitarios en el segundo.

En la ciudad encontramos algunos espacios residuales en los que no se desarrolla ninguna actividad, algunos autores los identifican como terrenos ociosos o huecos urbanos. Por tanto, el nuevo espacio público trata de integrar a estos terrenos ubicados preferentemente sobre, junto o cercanos a las vialidades principales de la ciudad. Siendo un elemento indispensable para el estudio de la integración de los huecos urbanos a los espacios públicos se opta por el uso de la fórmula de densidad media urbana, donde se contemplan la densidad, la población y la superficie para así analizar la unificación y consolidación del espacio público al entramado urbano.

La densidad media urbana (DMU) de cada municipio se obtuvo a partir de la densidad bruta (población entre superficie) de cada una de sus AGEB urbanas ponderadas por el tamaño de su población como se muestra en la tabla 1. Para el cálculo de la densidad media urbana se tomó la fórmula planteada por el INEGI. (SEDESOL, 2004) La densidad media urbana (DMU) de cada municipio se obtuvo a partir de la densidad bruta (población entre superficie) de cada una de sus AGEB urbanas ponderadas por el tamaño de su población como se muestra en la tabla 1. Para el cálculo de la densidad media urbana se tomó la fórmula planteada por el INEGI. (SEDESOL, 2004).

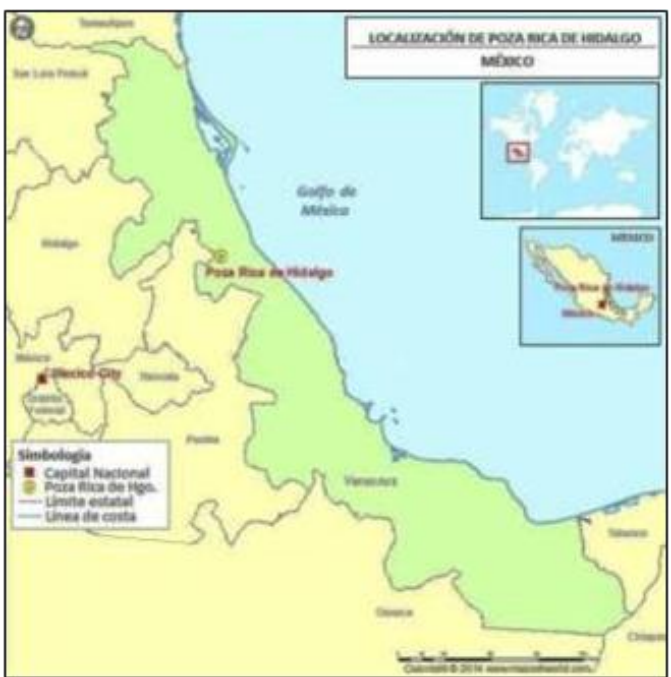

Figura 1. Ubicación de la ciudad de Poza Rica, Veracruz. Fuente: Mapa cortesía de www.mapsofworld.com

$$
D M U=\frac{\sum_{i=l}^{n} P_{i}^{2} S_{i}}{\sum_{i=l}^{n} P_{i}}
$$

Donde:

DMU

$\mathrm{Pi}$

es la densidad media urbana del municipio.

$\mathrm{Si} \quad$ es la superficie del AGEB urbana i.

Siendo Poza Rica el municipio central de la ZMPR llama la atención la mancha urbana extendida casi completamente en sus 64 hectáreas de territorio, con un amplio uso de suelo habitacional en la periferia donde se observa la proliferación de campos petroleros en uso o desuso actualmente, y que, estos últimos han sido fuente de espacios recreativos y deportivos gestionados por la administración municipal en turno ante las autoridades competentes en PEMEX. De tal forma se ha ganado espacios públicos para disfrute de la ciudadanía. De acuerdo a WRI México, los mexicanos cuentan con $1.2 \mathrm{~m} 2$ de áreas verdes por habitante en las ciudades muy por debajo de $\operatorname{los} 9 \mathrm{~m} 2$ que recomienda la Organización Mundial de la Salud.

Tabla 1. Población y Densidad Media Urbana por municipio de la ZMPR

\begin{tabular}{|c|c|c|c|c|}
\hline & Municipios & $\begin{array}{c}\text { Población } \\
\text { por } \\
\text { municipios, } \\
2010 \\
\end{array}$ & $\begin{array}{c}\text { Superficie }^{1} \\
\left(\mathrm{Km}^{2}\right)\end{array}$ & $\begin{array}{c}\text { DMU }^{2} \\
\left(\mathrm{hb} / \mathrm{ha}^{2}\right)\end{array}$ \\
\hline 49. & $\begin{array}{c}\text { Zona } \\
\text { Metropolitana } \\
\text { de Poza Rica }\end{array}$ & 513518 & $2,789.0$ & 63.4 \\
\hline 1. & Cazones & 23059 & 272.3 & 34.5 \\
\hline 2. & Coatzintla & 43106 & 277.7 & 71.3 \\
\hline 3. & Papantla & 152863 & $1,456.5$ & 50.9 \\
\hline
\end{tabular}




\section{4.}

Poza Rica

181438

64.1

70.7

5. Tihuatlán

80923

718.4

47.4

Fuente: CONAPO. Elaborado por el Grupo Interinstitucional con base en los Censos Generales de Población y Vivienda 1990 y 2000, y el Censo de Población y Vivienda 2010.

1. El dato de Superficie se obtuvo de las Áreas Geoestadísticas Municipales (AGEM), del Marco Geoestadístico Nacional 2010.

2. Densidad Media Urbana: El dato de superficie para el cálculo de la DMU se obtuvo a partir de las Áreas Geoestadísticas Básicas (AGEB) urbanas, de la Cartografía Geoestadística Urbana del Censo de Población y Vivienda 2010.

Una ciudad fortalecida, que avanza en su desarrollo se puede conocer a través de sus espacios públicos, si estos son confortables, seguros, conectados con las vialidades así puede interpretarse como una trama urbana competitiva. En la Agenda 2025 para el espacio público y la vida pública en México se declara que el espacio público es el activo más valioso con el que cuenta la ciudad para contribuir a su desarrollo estratégico considerando que más del $72 \%$ de la poblacional nacional habita en ciudades (Asociación Nacional de Parques y Recreación y WRI México, 2018), en lo cual concordamos debido a que el estudio se concentra en la generación del nuevo espacio público, y con ello, en su revalorización sin precedentes favorables a causa de la visión de habitabilidad y competitividad al tomar en consideración los beneficios en la mejora de la salud emocional, física y mental del usuario promoviéndose un efecto secundario en la mejor de la calidad visual, ambiental y del entorno urbano. En casos nacionales se pueden observar casos de peatonalización de calles donde el comercio local se ha beneficiado por la mayor visita a sus comercios.

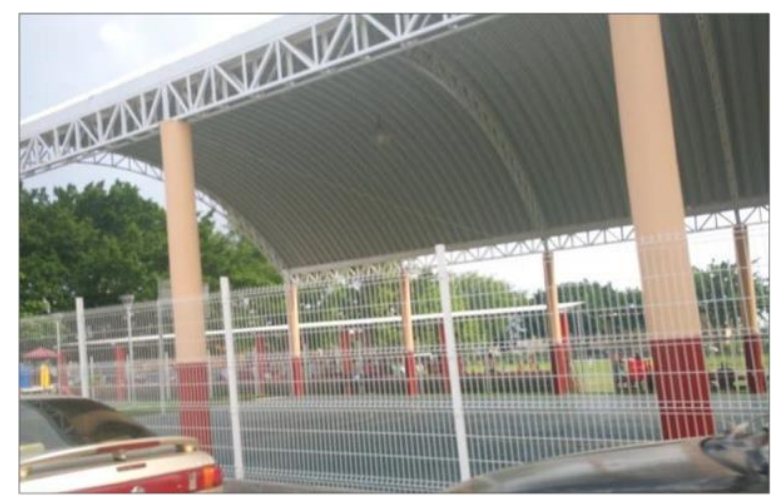

Figura 2. Domo sobre cancha de básquetbol como nuevo espacio público en el parque recreativo de futbol "Juan Chino" de la col. 27 de septiembre. Fuente: Blanca Inés Aguilar Frías. Marzo 2018

Con la información anterior, el presente análisis debe articularse en las propuestas y objetivos de los niveles de gobierno, y así, generar la posibilidad de creación de espacios vivificados, integrados a la estructura urbana, confortables, funcionales y seguros para todos. Con lo cual pasamos a la revisión de dos vertientes en un mismo espacio físico:

a) Mejoramiento físico.

b) Participación social y seguridad comunitaria.

Todos los proyectos para que adquieran el carácter de integral deben comprender obras y acciones de las dos modalidades. Esto significa que para el rescate de los espacios públicos se considera tanto la realización de obras de construcción, habilitación o rehabilitación de parques, centros deportivos, instalaciones artístico- culturales, entre otros, como la puesta en marcha de actividades orientadas a que la comunidad se organice y tome parte activa en tareas dirigidas a la prevención de la inseguridad y las conductas de riesgo.

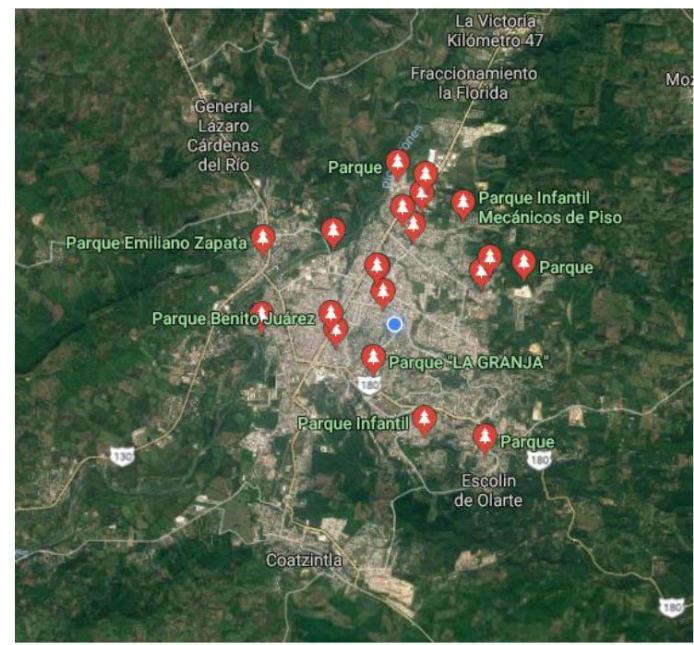

Figura 3. Ubicación de parques como espacio público en la ZMPR. Fuente: Cortesía de Google maps

En la figura 4 se observan ejemplos de espacio público tradicional intervenido, en todos los casos se trata de colonias periféricas del municipio de Poza Rica.

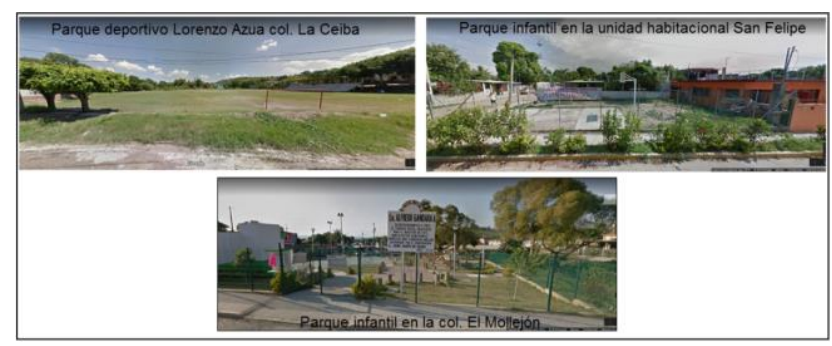

Figura 4. Espacio público tradicional: parques infantiles en colonias de periféricas de ZMPR. Fuente: Cortesía de Google maps.

\subsection{De pozos a parques urbanos}

El municipio central de la ZMPR lejos está de satisfacer la cantidad y calidad de instalaciones deportivas y culturales que compensen la demanda de la población metropolitana. Así la utilización de los anteriormente productivos campos petroleros dentro de la ciudad y actualmente improductivos, es una opción para el espacio público habilitado en parques urbanos.

Otro espacio residual en las colonias periféricas hábilmente aprovechado para cumplir con las estrategias planteadas para el cumplimiento de dotación de espacio público como en este caso, donde el parque urbano de la colonia Arboledas surge en el espacio residual y cercano a la vialidad de introducción a la colonia, como se puede apreciaren la figura 5 , siendo su característica un punto que fue relegado en el uso habitacional y segmentado de las vialidades de conexión a la colonia. Esta política pública responde al interés transformar los hábitos de desplazamiento e incentivar el uso eficiente del entramado urbano, muy especialmente de los espacios en desuso 


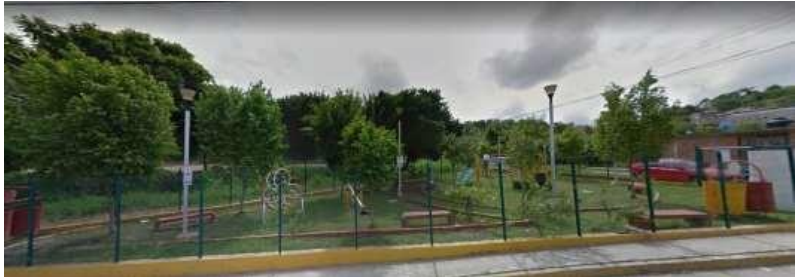

Figura 5. Espacio público ganado a través de un espacio residual en la colonia Arboledas.

El objetivo estratégico de la recuperación de espacios residuales es claramente referente a la salud: vigorizar y consolidar la creación de nuevos espacios públicos recreativos y deportivos para el combate al sedentarismo, inherente a este surge un segundo objetivo a mediano plazo como lo es el fortalecimiento a la seguridad, separando a la juventud de la ociosidad y proveyéndola de espacios aptos para su esparcimiento. La crisis ecológica, escalado a cada una de las urbes, se manifiesta en la crisis de espacios destinados a la recreación, deporte, cultura donde no se considera a la naturaleza como recurso sino como un bien factible de explotación urbana, para nuestro caso. Como señala Carrasco, la naturaleza es parte constitutiva de la sociedad, y por tanto, no es independiente ni objeto vendible (Carrasco, 2014). La fragmentación de las inversiones, la carencia de un plan maestro, la minimización de los beneficios disminuye la visualización de las administraciones que si invierten sus esfuerzos en la dignificación del nuevo espacio público.

\subsection{Movimientos internacionales:}

La agenda 2025 para el espacio público y la vida pública en México marca la generación y provisión de información relevante para el cumplimiento de la agenda y la generación de líneas de investigación con énfasis en los espacios públicos urbanos, espacialmente los parques. Existen proyectos en México y Latinoamérica que demuestran la importancia de concretar los procesos de planificación participativos donde se involucre la sociedad, donde se comparta el costo-beneficio, la eficacia y la sostenibilidad de los procesos. La morfología del espacio público y las condicionantes físicas, así como su uso marcan el sentimiento de apego al lugar, la identidad y la propia vida pública. (Colacios, 2017).

El aspecto de vivificar los espacios públicos desde un enfoque cultural-turístico renueva el beneficio por los mismos, ya que se espera una diversificación de la actividad económica que impacte de manera local generando prosperidad como en países que si cuentan con actividades habituales, programadas, con carteleras ya conocidas mes con mes y que con ello permite derribar las barreras de la monoactividad petrolera con que se conoce a la ZMPR.

\section{3.-Resultados y Discusión}

En Veracruz, las ciudades con orígenes en la industria petrolera, se cuenta con campos petroleros en desuso, algunos de ellos que han sido revitalizados en función del fomento a la convivencia social, el acondicionamiento físico y la recreación infantil, como el que se muestra en la figura 6.

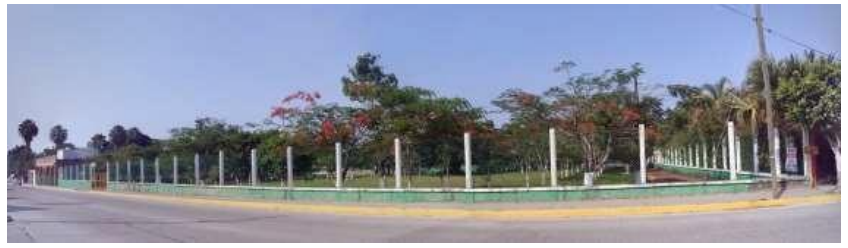

Figura 6. Campo petrolero en desuso adaptado como andador peatonal para los colonos del fraccionamiento Santa Elena. Fuente: Cortesía de Google maps

\subsection{De espacio público en desuso a parque urbanos.}

El espacio en desuso y vacío urbano en cuanto a su caracterización se da en el marco de la utilización del espacio público por la población. La valorización de estos espacios marca una constante: su uso futuro. Los posibles escenarios urbanos emergen como puntos factibles de encuentro social. Una de las dificultades para llevar a cabo el presente análisis es lo limitado de la información, la dificultad de concentración y medición de los datos, y con ello elaboración de la tipología de los espacios públicos dentro de la ZMPR. Para ser tomado como referencia tenemos el caso de los campos petroleros que se ubican a un costado de la Avenida Pozo 13 y Nezahualcóyotl, en el fraccionamiento Heriberto Kehoe, que se muestran en la figura 7 , y como puede observarse es un punto de interconexión vial dentro de la ciudad de Poza Rica.

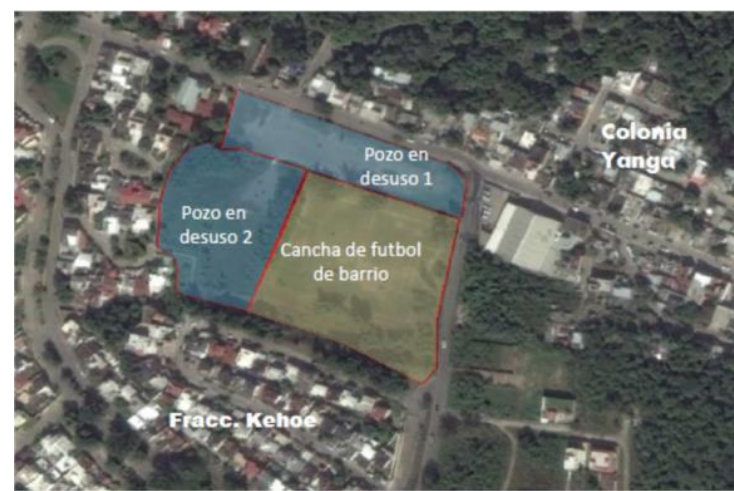

Figura 5. Espacio con posibilidad de intervención urbana, en rescate de campos petroleros en desuso dentro de la ciudad de Poza Rica. Fuente: Cortesía de Google maps.

Por lo tanto, es importante recuperar, dignificar y remodelar los espacios públicos (pozos adaptados) fomentando la participación ciudadana con proyectos incluyentes en la sostenibilidad y la cultura, con un uso adecuado de la escala urbana así como el respeto a los aspectos de diseño de accesibilidad universal y seguridad al usuario lo cual permitiría alcanzar una ciudad activa e incluye donde las personas puedan caminar, andar en bicicleta descansar dentro de su lugar de confort y con seguridad, siendo estos aspectos la tendencia a los nuevos conceptos de diseño de las polis. (Arredondo, 2017). Efectivamente, la densidad y la escala otorgan sentido a cada sector, área, barrio donde sus límites sean claros, se promueva la interacción cara a cara. Las cualidades con que se cuenta para que la densidad sea favorecedora es que prevalezca la diversidad de usos del suelo urbano por lo que es preponderante para no obstaculizar la diversidad de usos. (Talen, 2000).

\subsection{En la centralidad o el borde espacial}


¿Porque la periferia urbana? Por ser el laboratorio natural del ente provocativo para la adaptación de la ciudad. Pero la revolución no solo se observa en la periferia, en la centralidad consolidada de la ciudad también se encuentran dentro de la ciudad como tal, los pozos petroleros que ya no tienen producción y son factibles a una intervención, y es ahí donde surge el movimiento generador del espacio público en transición en etapa de consolidación. Los vacíos urbanos tienen un poder atrayente en dos vertientes: por su propia naturaleza y localización.

Lo conveniente es generar una red espacial de análisis de estos espacios urbanos con posibilidades de intervención para con ellos determinar su potencialidad de uso a espacio público (Abarca-Alvarez, 2017). Las condiciones de vida se vislumbran en el entorno discordante como expresión de crisis ambiental (Cantú, 2015) donde la apropiación del territorio eleva el valor del suelo urbano se percibe una necesidad imperante del adecuado uso del espacio público debido a que, no en pocas ocasiones, los espacios destinados para áreas verdes en las colonias son absorbidos para otros usos más rentables.

\section{Conclusiones}

Concebir el espacio público generado de los espacios residuales, o en desuso, de una ciudad favorece plenamente los puntos que se toman para la propuesta actual. Así este origen y evolución del espacio público en la ZMPR confirma el requerimiento significativo de lugares para la convivencia especializada de todos los grupos de ciudadanos de Poza Rica y sus visitantes.

Recordando que la ciudad se conoce a través de la calidad y cualidades de sus espacios destinados a la coexistencia donde confluyen ideologías. Con el estudio de este tema se ven beneficiados los habitantes de la ZMPR con la caracterización de sus espacios públicos, un adecuado uso de los lineamientos de diseño urbano ambiental y de sostenibilidad en cada punto a analizar, al igual que los resultados esbozados se apoyarían en un estudio de costo beneficio del proyecto, una reforestación adecuada y con estudio solar.

\section{English Summary}

The conformation of the new public space in the metropolitan area of Poza Rica.

\footnotetext{
Abstract

The large city generates commitments in the satisfaction of equipment and infrastructure and in terms of public and private spaces. The Metropolitan Area of Poza Rica (ZMPR) in its central municipality, the urban park, as equipment for the healthy development of modern city dwellers, is revealed as a scaffolding for the revitalization of community life avid in its attention to this type of space considerably scarce and with poor accessibility over time. Modern cities with intervened public spaces are positioned as interconnection nodes between the central points of the same, reconciling with them the integration to the urban framework necessary for the vivification and consolidation giving character and identity to
}

each area. The checkerboard of the city has gray areas in the neighborhoods, school areas and roads identified with potential use for these urban spaces with strong citizen participation to perform actions that interfere with children, adolescents and seniors to raise awareness of accessibility principles universal and sustainable design for safe public spaces. The urban park as a space of social expression houses the daily collectivity, the collective memory transforming itself from being considered only as the landscape space par excellence giving way to coexistence and modal transit. It is precisely the residual spaces where the consideration of sustainable design of the new public space is a priority

Keywords:

Public space, universal accessibility, sustainability, extensive city, metropolitan area.

\section{Referencias}

Abarca-Alvarez, F. J. (noviembre de 2017). Identificación de patrones espaciales del borde urbano mediante mapas auto-organizados de la centralidad de la red viaria. Revista Urbano(36), 18-29. Recuperado el 3 de Febrero de 2018

Arredondo, I. A. (2017). Elespacio público de los puente peatonales: lecciones del BRT de Bogotá. Revista Urbano(36), 104-114. Recuperado el 3 de Febrero de 2018

Asociación Nacional de Parques y Recreación y WRI México. (2018). AGENDA 2025 para elespacio público y lavida pública en México: un llamado colectivo a la acción (Primera ed.). Mérida, Yucatán,

$\begin{array}{llllll}\text { México: } & \begin{array}{l}\text { ANPR. } \\ \text { mayo }\end{array} & \begin{array}{l}\text { Recuperado } \\ \text { de }\end{array} & \text { el } & 28 & \text { de }\end{array}$
http://wrimexico.org/sites/default/files/AGENDA2025\%20EPVP\%20ME XICO_VF.pdf

Cantú, R. (2015). La sostenibilidad del centro histórico. México, México: Plaza y Valdés. Recuperado el 30 de enero de 2018

Carrasco, R. (2014). Territorio apropiado en la insustentabilidad. Contribuciones críticas. (1ra. ed.). (PyV, Ed.) México, México: Plaza y Valdéz. Recuperado el 21 de Enero de 2018

Colacios, R. y.-A. (2017). Uso e interacción social en el espacio público: el caso del polígono de vivienda Sant Cosme, Barcelona. (36), 66-77. doi:https://doi.org/10.22320/07183607.2017.20.36.06

INEGI. (2007). Instituto Nacional de Estadística y Geografía. (inegi, Ed.) Recuperado el 17 de mayo de 2018, de http://www.beta.inegi.org.mx/app/buscador/default.html?q=areas+recreati

Michel, B. G. (2005). La historia de la ciudad...es la de sus espacios públicos (Vol. XXVI). Cuba, Cuba: Redalyc. Recuperado el 27 de mayo de 2018, de

http://revistascientificas.cujae.edu.cu/Revistas/Arquitectura/Vol-XXVI/12005/07-15- Lahistoriadelaciudad....pdf

OMS. (2010). Urban planning, environment and Health: from evidence to policy action. Organización Mundial de la Salud. Obtenido de http://www.euro.who.int/data/assets/pdf_file/0004/114448/E93987.pdf?ua $=1$

Perahia, R. (2007). La ciudad y su espacio público. Porto Alegre: Universidade Federal do Rio Grande do Sul. Recuperado el 27 de mayo de 2018, de

http://www.ub.edu/geocrit/9porto/perahia.htm

SEDESOL. (2004). Delimitación de las zonas metropolitanas de México. México, México: SEDESOL. Recuperado el 15 de mayo de 2018, de http://www.conapo.gob.mx/work/models/CONAPO/zonas_metropolitanas 2000/01.pdf

Talen, E. (2000). Measuring the public ealm: A preliminary assessment of the link between public space and sense of communnity (Vol. 17). Journal of Architectural and Planning Research. 